\title{
Introduction: Symbolic Imagination
}

Jan Ch'iu said: It is not that your Way does not commend itself to me, but that it demands powers I do not possess. The Master: He whose strength gives out collapses during the course of the journey (the Way); but you deliberately draw the line.

The Analects of Confucius, trans. Waley, 6.10

There is an image well known among Tantrics of the last century, though not common now; one may call it an image for want of another descriptive term, for, in fact, it consists of no image at all-or, rather, it is an image of nothingness-since what is imaged is only an open frame, to which are attached a pair of arms and sometimes legs and ears (Fig. I). As in some Eskimo masks, we find attributes attached to a periphery, only in this case that periphery borders an empty space. The empty frame, standing for all that is possible and impossible, represents for certain Hindus the Goddess as Supreme Void, a void that is specified by the frame that marks it out. Like the house in the Tao Te Ching (chap. II), in which windows are defined by the empty space they enclose, the frame marks the bounds: it draws the line, it sets the limits within which meaning must manifest itself.

Every year on the Feast of the Ascension, the vicar of the Church of St. Mary the Virgin, a parish church of Oxford and the University's first building, ventures forth in solemn procession to a number of points that mark the boundaries of the parish. At each, whether it be in the quadrangle of an ancient college or in what is now the ladies' apparel department of a modern clothing store, a ritual known as "beating the bounds" is enacted. A prayer and a song by the choir begin and end the actual beating, in which the spot, already designated by a cross, is marked by thrashing it repeatedly. Why the bounds are beaten no one can precisely say (but compare Needham 1967), any more than 
we can now understand a similar "bumping" of the bounds that took place in Bristol up until the turn of this century; but the practice of ritually marking boundaries may be glimpsed in the earliest evidence we possess for what may be called human culture.

Both the above examples - the Tantric frame and the beating of the bounds-demonstrate how otherwise ordinary space may be delineated in such a way as to create a context wherein real actions take on extraordinary meaning. In the second case, the real character of the actions appears startling precisely because they mark otherwise unnoticed points in the real world; in the former-the few inches of bronze frame-a space no less real is set out despite our physical incapacity actually to occupy the tiny bit of air the frame marks out or the section of reality it visually frames. With these two examples, we perceive the difference between image and imagination, between those static objects that stand for something else and those stages upon which objects become marked images through imaginative acts. The distinction is clear, yet it is one that ritual forever attempts to resolve. In ancient China (Waley 1938, 64),

the word $l i$ ("ritual") is expressed in writing by a picture of a ritual vessel. The original meaning is said to be "arranging ritual vessels"; and this may very well be true, for it appears to be cognate to a number of words meaning "to arrange in proper order," "to put in sequence," etc.

The ordering of vessels, thus, provides ritual meaning, so much so that the breaking of such vessels is, as one would expect, symbolic of chaos, much as in Hindu tradition, in which smashing a pot symbolizes loss of life in certain death rites. Naturally, part of the connection between a pot and a human life derives from what Tylor understood as the great principle of sympathy. In southern India one still ties the afterbirth of a calf to a milky-sapped tree to encourage it to give much milk in later life (Fig. 2). But sympathy here is not simply the result of recognizing that such trees are great containers for the life-giving sap (rasa) that is in everything that lives; it is part of the greater recognition that what we call symbols must be contextualized in order to achieve meaning. In this respect, the marking of boundaries results in no less an objective image than a line drawn on paper or a bronze statue of Śiva. However, what distinguishes the objective image from our 


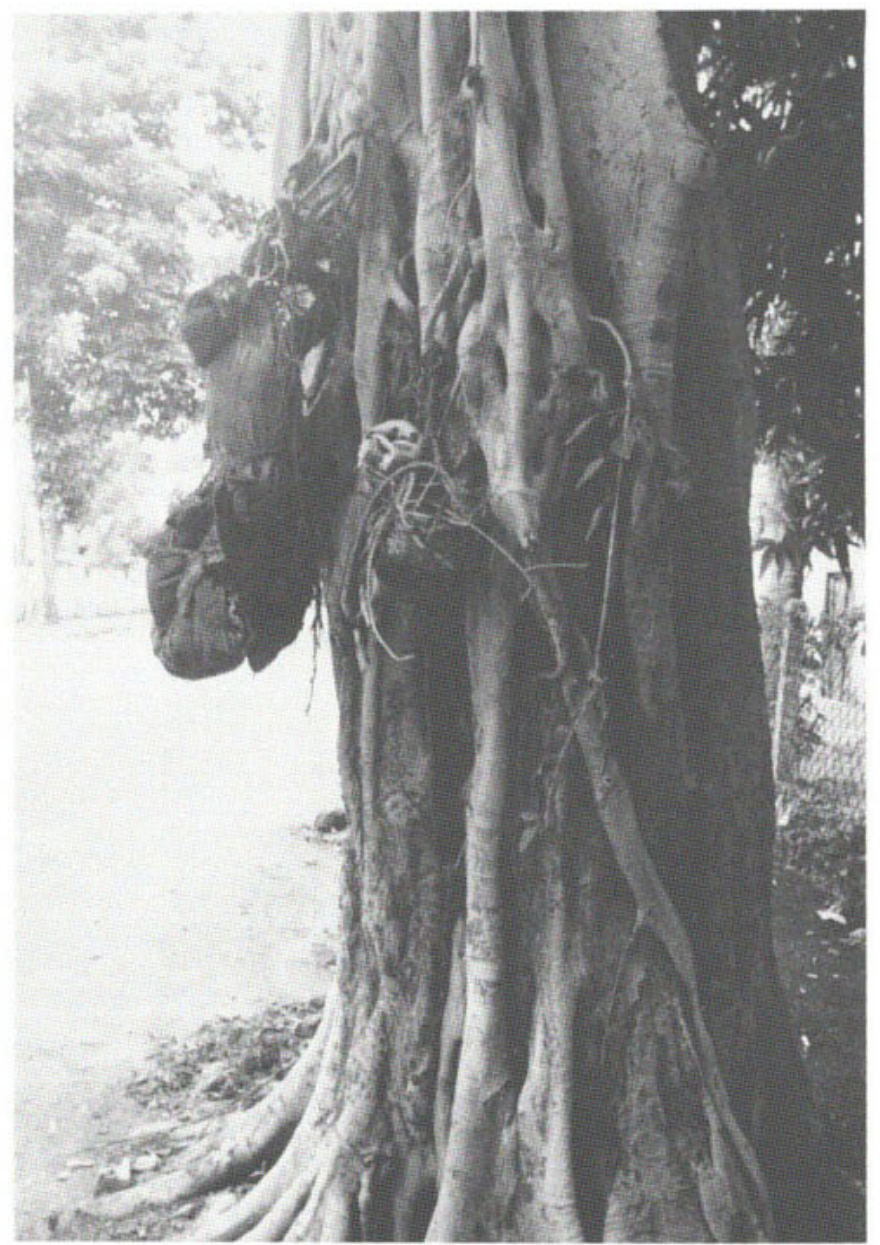

FIG. 2. Cows' afterbirths attached to milky-sapped tree (Madras, India, 1985): in the interest of securing a plentiful production of milk, water buffaloes' afterbirths are wrapped in bundles and attached to a tree known for its copious supply of milky sap. (Photo: author) 
traditional conceptualization of the process of imagining is that the drawing or statue is meant to stand for something else in all circumstances except when those images are ritually charged. In ritual, they may actually become something else if that other thing-god, demon, or other personified force-can be compelled to become identified through them; while, conversely, the ritual marking of boundaries sets the limits within which real objects become supernormal, where events take place that, otherwise, might have passed as a normal part of the real world or might even have gone unnoticed. The one imagines through the image of some thing; the other through the frame that isolates that thing. Between the two-between object and context-is the ritual activity that marks the connection; it is within ritual that objective images become contextualized and contexts become imagemaking or imaginary.

Thus, in ritual, objects and actions become contextual and connotative. They "identify" insofar as they demonstrate some absolute likeness existing between two things-a frame and the Void, a vessel and the world-and they represent insofar as each image is understood to be distinctive, a re-creation, something else. A symbol is, therefore, something that both stands in place of and represents something else while, at the same time, partaking of that other thing; and symbolic space provides the stage, the container, the vessel that delimits the ways a symbol may be manipulated. That a stage may be a vessel, or an empty frame the Void, is not merely a metaphorical correspondence, for the microcosmic symbol stands in sympathy with the larger phenomena that constitute what we call "reality"; the symbol becomes the basis for other sympathetic relations, so much so that actions occurring in the real, actual, or architectonic world may be inseparable from their symbolic content. Such inseparability is evidenced in nearly all images we recognize as symbolic, and it is in the very nature of symbolic images that they encourage this inseparability both through their archetypal character and their adaptability. When a Hindu is charged to pick out with the twigs of a milky-sapped tree the bones from a cremation pyre, the original image of the all-permeating fluid rasa is not merely certified; the action reiterates, through yet one more example, the correspondence between the symbol and events in the real world.

In the realm of symbolic language, the process of drawing a cor- 
respondence between a symbol and its context may be clearly observed in the construction and modification of ideographs. In refined and long-standing ideographic languages such as Chinese, the actual images may be transformed so greatly as to have made their original shapes unrecognizable, or, indeed, the shapes may have come to indicate phonetic values rather than visual ones. Difficult though recovering the processes may be for philologists, the mechanisms themselves may be easily observed elsewhere. I was astonished some years ago to find how readily children from about their fourth to their sixth year of age are able to create and sustain a complex symbolic vocabulary. Theirs is an ability that not only, once developed, can be maintained, but one that can be only very crudely learned if begun a few years later-after, that is, they have been conditioned to attach phonetic values to a written script, and after they have proceeded beyond the developmental stage associated with what Vygotsky called chaincomplex formation. At four years of age, a child is eager and ready to experiment with the fluid sympathy it perceives to exist between objects and their environment.

The experiment I employed is quite simple and easily replicated. Each child is given a piece of paper on which is drawn some arbitrary form. A circle, a triangle, even a line will do, though so will symmetrical shapes; at any rate, each child will before long be developing his or her own typology. One need only introduce the idea that a specific shape can have whatever meaning the child perceives it to have. Little encouragement is required for children to tell one what the form "is." The trick comes of their discovering that the shape can-like the empty Tantric frame or an Eskimo shaman's mask-become an image upon which attributes may be hung, and that the form of this image need not be disassembled before a new type is created. The child proceeds, to use the Chinese metaphor, to set up ritual vessels, to place similar modified shapes in sequence. What results is a story (Fig. 3) that the child has invented; but what is more significant is that the story can be read, developed, and modified. It can be "read" by one child to the next; it can be put away and its meaning recovered at a later date. The child has discovered a means of recording, conveying, and, most important, recalling a complex series of ideas. Without years of schooling the child has discovered the pleasure of reading and writing; it has de- 

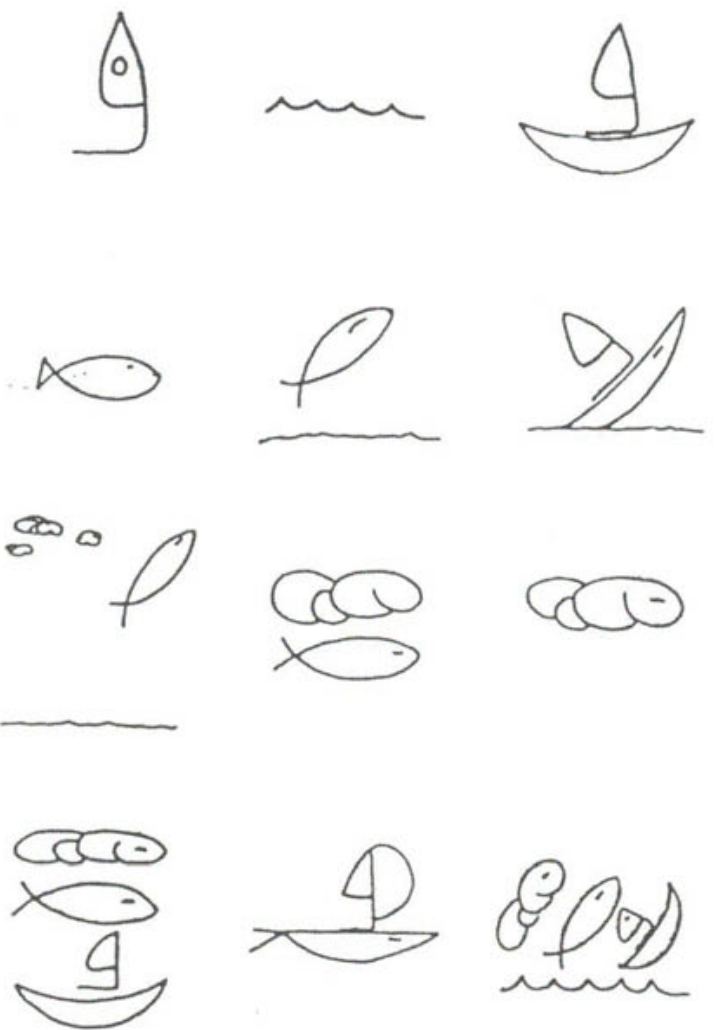

FIG. 3. This ideographic story, in which abstract images become sails, fish, and clouds, was developed in conjunction with preschool children, and illustrates how able they are to invent ways by which conceptual categories may be merged, conflated, or transformed. (1973; author's collection)

veloped a poetic imagination that will form the basis of a later love for the manipulation of language. The child has suddenly and almost spontaneously discovered how something may stand for something else. At this point in its development, the mind is entertained by the knowledge that symbols and events-and, indeed, all things microcosmic and macrocosmic-may be connected. It will be some time 
before the child is resigned to the cultural demand that divisions and distinctions be part of knowledge, that nothing can be known, as Aristotle once said, except in its parts. For the moment, at least, children are still delighting in Aristotle's equally profound realization that what makes the world one will also be what makes a man.

It is with this last realization that the following five essays both begin and end; in fact, the penultimate essay literally ends with this dictum, as did the final paragraph of my Masks, Transformation, and Paradox, the study from which these essays stem. However, before saying more about the individual essays that make up this collection, it is necessary to make one point with regard to the fundamental connection between images and imagination; for a symbol is not only a microcosmic reflection of something macrocosmic, not just a distillation. It is, as importantly, an irreducible thing, the smallest atomic unit to which the macrocosmic may be reduced. It is the graphic point, the bindu of Tantrics and yogis, beyond which reduction of a greater image becomes impossible. Conversely, it is also the point where visualization begins. It marks, in other words, the birth of imagination. The moment at which we abandon a holistic view of the universe is precisely that moment at which visualization becomes impossible, where "imagination," in its strictest etymological sense, disappears; and the moment we let words-that is, not visual impressions-qualify as "symbolic," we make our first move away from what will qualify, in what is to follow, as "symbolic thought." The ability to visualize symbols, therefore, is directly commensurate with their ability to function in some atomic capacity. This is precisely why visualization is essential for symbols, and why the symbolic imagination becomes impossible in a world view in which the absolute correspondence between microcosmic and macrocosmic is not sustained. One obvious example of this loss of symbolic imagination occurs with the advent in science of the quantum-mechanical view, which Einstein so aptly criticized for its disjunctive way of treating phenomena and its consequent incapacity either to state its problems simply or to visualize them. There are many other examples of this devisualizing process, but what is important to emphasize is the exact correspondence between the loss of imagemaking capabilities, of imagination, and the abandoning of an atomic, $\mathrm{microcosmic/macrocosmic} \mathrm{world} \mathrm{view.}$ 
The responsibility for explicating other modes of thought that are focused on a systematic and structural coherence between microcosmic and macrocosmic relations has frequently devolved upon anthropologists who have committed themselves, often tirelessly, to the systematic study of alternative categories of thought. The origins of this interest are, of course, much older than social anthropology itself, but at least from Durkheim onwards the discipline has been largely modeled on the notion that the "systematic combinations of collective representations" (Needham 1972, 158) are social facts and that, therefore, the higher forms of imagining and ideation are largely social phenomena, particularly when modes of thought function to elaborate categories of thought that provide a unique perspective on how images might be actualized-how, that is, human self-awareness relates categorically to the larger framework that we call the objective world. Thus, while Durkheim wished to distinguish the role of the social sciences from the domain of psychology, it is also the case, as Beidelman points out, that "it remains difficult to separate some forms of sociology from a 'higher' form of social psychology" (1986, I0) and that, in this sense, the systematic study of modes of thought involves the use of imagination as a kind of moral exercise (ibid., 2). ${ }^{1}$

What, we may ask, do ontological perspectives about object relations have to do with morality? As Simmel has shown, objectivity in and of itself may be the primary determinant of social freedom, since "the objective individual is bound by no commitments which could prejudice his perception, understanding, and evaluation of the given" (1950, 405). Conversely, in cultures for which one's perceptions are regularly guided by rules that govern microcosmic/macrocosmic connections, one's relations to objects are, likewise, more carefully established and nurtured. For this reason, anthropology has seen not only a resurgence in attention to the study of the social role of objects, but particularly an interest in the way that the "gift" (le don)-in the sense of something that is an extension of the giver-may be distinguished from commodities (i.e., from things that are "depersonalized" to the

I. By "moral," Beidelman also means mos, i.e., "a way of comporting oneself" (ibid.), a way of acting, of being a social person. 
extent that they acquire an absolute value that is "replaceable"). ${ }^{2}$ Moreover, when objects thus considered become vehicles for establishing the connectedness of things, rather than entities that by definition must be distinguished and separate, one can readily perceive how they become essential to culturally significant notions of hierarchy, and to notions, moreover, of how hierarchy may function in the development of canons, in the establishment of the very idea of culture and what distinguishes "our" culture from "theirs." In such a limited study as the present, it is possible only to intimate how these central and intimidating problems of the discipline are related, and many of them, therefore, cannot be treated comprehensively; but it is my hope that the economy I have tried to introduce into an enormous topic will at least suggest how modes of thought that are particularly alien to postEnlightenment civilization in the West can remind us that our concepts of culture-and, therefore, of the foreign-are intimately connected to how we envision symbolic thinking or how we understand it to function.

The following collection consists of essays in which symbolic correspondences are discussed with reference to notions of the foreign. In them, the "absolute likeness" that is part of identity is presented as the complementary opposite of a given notion of the foreign. The third and fourth essays show how specific symbols may be employed in seeking out, identifying, and encompassing the foreign, and how specific symbolic forms are part of the same "image-making faculties"to use again Aristotle's categories - through which we "identify" ourselves. That we understand ourselves by looking at the foreign is noth-

2. Of special significance here is the recent work of Weiner (1983, 1985); but see, among others, Appadurai (1986) and Stocking (1985), and references therein. See also my discussion of the relation of objects to modes of thought (1986). Note, especially, how the "replaceability" of commodities is mirrored by the shift in notions of identity from a focus on "selfsameness" to "the absolute likeness of two or more things" - things that may stand in for one another and that are, therefore, "replaceable" (i.e., objectified). That such "commoditizing" does, indeed, have a moral dimension is, paradoxically, apparent in the degree to which we feel the need to introduce "morality" as a regulatory concept in contemporary cosmopolitan contexts. By contrast, I temember once hearing the current Dalai Lama describe Tibetan Buddhism as "amoral." What he meant, of course, was that Buddhism offered a release from self-conscious considerations that were explicitly about morality by providing a macrocosmic symbolic construct in which morality was implicitly a part of all conscious and subconscious activity. 
ing new, but it is my hope to show in these essays how specific symbols are at the foundation of our capacity not only to "imagine" the foreign, but to form an "image" of and for ourselves, a correspondence, an absolute likeness, an identity.

By contrast, the first two essays focus on a converse anthropological point-namely, that contemporary art must be willing to alter its traditional notions about artistic "identity" if it is to escape the loss of "imagination" that has characterized (as one now often hears) so much of modern art. My argument here is not that the art of the present has become too detached or avant-garde; quite the opposite. I argue that whatever loss of "imagination" or deterioration of the image-making faculties there may be is the result of the essentially conservative definition of the personality of the modern artist that modern society supports-a definition that, on its own terms, precludes artists from being innovative and, thereby, makes impossible their living up to the radical demands necessitated by a sustained and rigorous commitment to the avant-garde. I wish to show hereby not that the radical theses set down by earlier manifestations of the avant-garde in the late nineteenth and early twentieth centuries cannot be fulfilled, but rather why they have yet to be fulfilled. If I offer a view of contemporary art that represents it as too conservative, I do not do so as a disparager, but as an admirer and participant observer, as one who foresees a time when artists can gain an intellectual preeminence that they have, for various reasons, been denied and that, in turn, they have denied themselves.

Having alluded-albeit briefly and even, perhaps, cryptically-to the agendas of this book, it seems only fair that I should also state in more specific terms what motivated me to organize these five different arguments under a single title. The diverse domains of inquiry that form the subject of these five chapters and the Epilogue fall into two intellectually distinct groups. The first of these (made up of Chapters I, 2, and 5, and including the Epilogue) sets out an argument about certain ways of imagining that are both largely neglected in the theoretical literature and relatively unexplored in those creative domains, or "art worlds" (to borrow Becker's words), that we sanction at the level of culture. In these three chapters (and in the Epilogue) I am largely arguing that there are specific techniques of knowing and of self-knowledge that we regularly mystify and, hence, "alienate"; and, 
furthermore, that these realms are, at least in part, knowable once we make the effort to demystify them by agreeing to approach them experientially, or, rather, once we realize the mystical potential in the ordinary, to paraphrase Goffman. I argue that, as a culture, we set up rules that proscribe any experiential venture beyond what is already known, and that we do so by distancing ourselves (intellectually, linguistically, emotionally) from those avenues that are actually quite close and readily available. I am not, therefore, treating the subject of "symbolic anthropology" as it is traditionally understood-that is, as a form of sociocultural anthropology that has evolved over the past few decades out of the work of linguistic theorists such as Pierce and Saussure. Rather, I am attempting to isolate domains of experience that are not as distant to us as our cultural canons encourage us to believe, and that, moreover, need not be as mysterious as the combined forces of rationality and Cartesian positivism suggest.

Though culturally disparaged, some of these experiential domains are, indeed, readily accessible to the inquiring mind, even though those that are closest to us are regularly denied intellectual status. Thus, while it may be the case, as Mary Hesse says of metaphor, that we must construct "a revised ontology and theory of knowledge" (I984, 4I), the "radical challenge to contemporary philosophy" (ibid.) posed by such modes of thinking is not, as some philosophers would have it, only the result of how different are such ways of thinking; rather, the real challenge comes of trying to imagine these processes as having value comparable to other forms of thinking to which, as a culture, we have given prejudicial treatment. The challenge, in other words, is to credit readily accessible ways of thinking that are simply different. The bricoleurs and idiots savants are there before us whether or not we give them serious attention; and their ways of embodying, of understanding, are, I will try to show, at least partially accessible to us and may even come to be "known" through a concerted attempt to move within a meaningful social space to which we have been sensitized. I am talking here not only about "participation" in the classic sense first articulated by Lévy-Bruhl; nor is my argument simply one about "knowing through doing." Rather, I am arguing for certain forms of knowing that come to life only when richly meaningful social spaces are created-spaces in which new forms of experience can be ap- 
proached and otherwise unrelated things can be formally juxtaposed or superimposed in ways that are creative.

It is also important to note that the dangers of engaging other ways of thinking are, as anthropologists well know, quite real, especially when they are not socially contextualized: it is no accident that any discussion of "modes of thought" regularly focuses on the symbolism of the left, on things often bizarre, and on mental states that are frequently unstable. These dangers are, moreover, evident in the degree to which culture steers us away from such modes of thinking by "alienating" them and identifying them exclusively with the dangers of losing one's self. However, if it is true, as I argue in what follows, that the ways of thinking described in this book are closer to us than we often think them to be, we need only look before us before taking the first steps. Moreover, if we are to believe what anthropology has taught us about rites of passage, it may also be the case that the very symbolic forms that empower such experiences can, if carefully constructed, also protect those who experience other, new modes of thought by offering a bounded and ordered symbolic domain, an experimental social context for exploring different instruments of cognition. Within such arenas, new images can be created through the connection (conjunction, juxtaposition, superimposition) of things that are otherwise unrelated. The results are not mere imaginary "collages," but images whose creative potential is not predetermined; and the imaginative character of, literally, seeing things differently becomes not merely a destabilizing experience.

The role of Chapters 3 and 4 is meant to be exemplary. They not only, therefore, offer historical studies of "otherness," but provide two specific and detailed examples of how a new approach to an old problem was generated by what was in my own initial thinking an unlikely juxtaposition of images or ideas in a regulated symbolic context. These chapters are not only arguments about theories of the foreign but the outcome of a particular method that works from a regulated context to a possible thesis, rather than from fact to fact. (Indeed, the thesis in Chapter 3 began as a rumination over a false etymology, while the dominant image discussed in Chapter 4 literally took shape at an introductory art lecture during which I imaginatively superimposed an image I had been reading about the day before on the one projected by the lecturer.) 
Thus, whether my conclusions in these chapters are right or wrong, at least I hope to convey that the method (and it is a method) by which the solutions were arrived at had, at least initially, less to do with deductive reasoning than it had with the unlikely comparison of two things bearing in the beginning a similarity that could only have been described as "superficial." My comparison of the Greek Gorgon with Indian demons in Chapter 3, and of the Piazza San Pietro with a Gnostic intaglio in Chapter 4, both originated in the technique of submitting elements to a comparison, despite their unlikeliness on anything but a superficial level; indeed, I will even argue in this book that it is precisely the superficiality of each example that made possible a reenvisioning of what the symbols considered might actually mean. In each case, I offer a novel solution to an old puzzle; whether, therefore, either solution finds general acceptance is, on one level at least, secondary to the fact that each provides a demonstration of how a symbolic context governed by an attention to microcosmic-macrocosmic continuity establishes a place for an active hallucination to be contextualized.

In the spirit of just such a juxtaposition, I should, first of all, like to express my deep thanks to Jiří Kolár for providing many years ago the arresting image, La Soif du passé, for the cover of this book. At a time when I was myself actively engaged in the creation of a kind of art characterized by unlikely juxtapositions, the discovery of his work was, as it still is, an invigorating experience. But I should also like to thank at this juncture the many artists I discuss herein; for, regardless of whether or not what I have to say about their efforts is flattering, they have for the most part been extraordinarily kind and cooperative in providing photographs of and additional information about their works.

Among those who have directly influenced the critical content of this book, I would like to thank Gregory Nagy for inviting me to formulate my views on the Perseus-Gorgon myth for his seminar on myth and myth theory sponsored in 1984 by the National Endowment for the Humanities. I would also like to thank the other members of the seminar, and particularly John Hamilton, S. J., for their enthusiasm and interest in what has now become the third chapter of this book. This chapter has also benefited by the suggestions of many others who have read it in draft form. Of them I would particularly like to thank Walter Burkert, Diskin Clay, Alan Entwistle, Nanditha Krishna, 
xxviii Foreign Bodies

Glenn Markoe, Richard Seaford, and Jean-Pierre Vernant. For the first and second chapters, I am most grateful to the Belgian artist Constant Lambrecht for weaning me away from postgraduate work in philosophy and toward the artist's life I followed for some years before deciding to think and write about art cross-culturally. Jonathan Benthall and Andrew Duff-Cooper also deserve thanks for their thoughtful comments on this part of the book. For the fantastic thesis offered in the fourth chapter, I take full responsibility, though I should like to thank Leopold Ettlinger, John Hunisak, and Irving Lavin for criticisms and the encouragement required by anyone who undertakes a topic as baroque as Bernini's iconography. Here, credit is also due to the editors of Res for publishing this essay in an earlier form. To the American Institute of Indian Studies, I owe thanks for inviting me to India to study the role played by Indian religion and art in traditional Balinese notions of the foreign. While that research will become part of my ongoing work on traditional Balinese culture, the leave of absence from my teaching duties provided me with the opportunity to present my interpretation of the Perseus-Gorgon story to several audiences in India; indeed, the enthusiasm of these audiences, as much as any of my own research, provided the catalyst to pursue the publication of this work. To Middlebury College I am grateful for providing the release time to take up the American Institute of Indian Studies fellowship. Not only was my work in India invaluable for revising Chapter 3 , but the fellowship also provided me with the opportunity to write this Introduction, to organize the essays into book form, and to experience personally several new notions of the foreign. Another fellowship, from the Andrew W. Mellon Foundation, made it possible for me to participate in a faculty seminar at New York University on Marcel Mauss and symbolic exchange, and I would like to express my thanks to Annette Weiner for inviting me to participate in that 1986 seminar and for the several discussions we have shared about our work since then.

Though there are many others to whom I owe thanks, Cynthia Atherton, R. H. Barnes, Judy Barringer, T. O. Beidelman, Hubert Decleer, Wendy Doniger, James Fernandez, Eric Frank, Ashraf Ghani, Byron Good, Mary-Jo Del Vecchio Good, Guy Hedreen, David Kessler, Arthur Kleinman, Donald Lopez, Emily Martin, Partha Mitter, 
David Nugent, John Palmer, James Peacock, David Pocock, and Paul Stoller deserve special thanks for their comments on various parts of the manuscript, and my copyeditor, Paul Psoinos, deserves a double thanks, as it were, for his two careful reviews of the typescript. For research assistance I am grateful to Lyssandra Barbieri, Elisa Barucchieri, Kara Hordlow, Lou Murrin, Stephen Plum, Michael Rhum, and Fleur Laslocky and the staff of Middlebury College's Interlibrary Loan department, and likewise to Megan Battey and Guanlong Cao for photographic work. To Francis Huxley, Rodney Needham, and my wife, Elizabeth, I am especially indebted-not only for urging me to bring these studies together under one cover, but for encouraging me to pursue topics on which, despite their disparities, I was intuitively led to reflect. 
